\title{
FISIOTERAPIA E TERAPIA OCUPACIONAL
}

\section{A ATUAÇÃO DA FISIOTERAPIA NA PREVENÇÃO DE ÚLCERAS DO PÉ DIABÉTICO}

\author{
DOI: http://dx.doi.org/10.31072/rcf.v9i1.575 \\ THE PHYSICAL THERAPY APPROACH TO PREVENT DIABETIC FOOT ULCERS \\ Janaina Santos Sousa*1; Nayara de Almeida Consoline*2; Paula Daiane Anízio*3; Patricia \\ Morsch4; Diego Santos Fagundes ${ }^{5}$.
}

\begin{abstract}
RESUMO: O portador de Diabetes Mellitus necessita de cuidados preventivos em função das complicações dessa patologia. Esse trabalho tem como objetivo descrever a atuação da Fisioterapia na prevenção de úlceras de pacientes com pé diabético. Foi resultado de uma revisão de literatura, realizado por meio de pesquisa na base de dados Biblioteca Virtual em Saúde utilizando os descritores "Fisioterapia; úlcera, pé diabético". O diabetes pode levar a complicações severas como o risco de desenvolver úlceras do pé diabético, culminando em possível amputação. A fisioterapia atua com ações preventivas na rotina do diabético exigindo conhecimento e preparo adequado desse profissional.
\end{abstract}

Descritores (DeCS) ${ }^{6}$ : Fisioterapia. Pé diabético. Úlcera.

\begin{abstract}
Individuals with Diabetes Mellitus needs preventive care due to complications of this disease. This study aims to describe the role of physiotherapy in preventing ulcers in patients with diabetic foot. It was the result of a literature review conducted by research in the BVS (Virtual Health Library) database using the keywords "Physical Therapy; ulcers, diabetic foot." Diabetes can lead to severe complications such as risk of developing diabetic foot ulcers and also amputation. Physical therapy works on preventive actions in diabetic patients' routine which requires knowledge and training of this professional.
\end{abstract}

Descriptors: Physical therapy specialty. Diabetic foot. Ulcer.

\footnotetext{
* Igualdade de autoria.

${ }_{1}^{1}$ Acadêmica do Curso de Bacharelado em Fisioterapia da Faculdade de Educação e Meio Ambiente (FAEMA). E-mail: janayna_ka@hotmail.com. ORCID: https://orcid.org/0000-0003-3374-1836;

${ }^{2}$ Acadêmica do Curso de Bacharelado em Fisioterapia da Faculdade de Educação e Meio Ambiente (FAEMA). E-mail: nayara.consoline@outlook.com. ORCID: https://orcid.org/0000-0001-6601-960X;

${ }^{3}$ Acadêmica do Curso de Bacharelado em Fisioterapia da Faculdade de Educação e Meio Ambiente (FAEMA). E-mail: paula-anizio@hotmail.com. ORCID: https://orcid.org/0000-0001-6842-1440;

${ }^{4}$ Doutora em Gerontologia Biomédica pelo Instituto de Geriatria e Gerontologia da PUCRS. Coordenadora do Curso de Fisioterapia da Faculdade de Educação e Meio Ambiente - FAEMA. E-mail: patriciamorsch@hotmail.com. ORCID: https://orcid.org/0000-0001-7186-8219;

${ }_{5}^{5}$ Doutor em Farmacologia e Fisiologia pela Universidad de Zaragoza - Espanha. Professor do curso de Fisioterapia da Faculdade de Educação e Meio Ambiente (FAEMA). E-mail: diegofagundes@hotmail.com.

6 Vide http://decs.bvs.br.
} 
O Diabetes Mellitus (DM) é uma das maiores causas de morte no mundo. É uma doença que acomete o sistema de consumo da glicemia, favorece a alta produção hepática de glicose, a desregulação na produção intestinal de hormônios incretínicos, bem como a diminuição na secreção de insulina pelo pâncreas $^{(1)}$.

Uma das complicações crônicas da DM são as úlceras do pé diabético, caracterizadas como lesões cutâneas com perda de epitélio, podendo acometer os tecidos profundos e até mesmo estenderem-se pelo membro inferior ${ }^{(2)}$.

Alguns fatores como andar descalço, o uso de sapatos apertados, cortes inadequados das unhas e pequenas dermatoses são prejudiciais aos portadores de Diabetes ${ }^{(3)}$. Segundo a Associação Americana do Diabetes, é de extrema importância a atuação da equipe multiprofissional focada nos cuidados do pé diabético para proporcionar melhor qualidade de vida aos portadores de DM, diminuindo os riscos de complicações ${ }^{(4)}$.

Estudos sobre a atuação da fisioterapia nessa patologia são recentes, e a intervenção mais utilizada em casos considerados leves é a prevenção. Já em casos mais graves são utilizados métodos específicos como placas de hidrocolóide, drenagem, cuidado com posicionamento, entre outros ${ }^{(5)}$. Sendo assim, essa revisão teve como objetivo descrever as condutas fisioterapêuticas na prevenção de úlceras em pacientes com pé diabético.

O presente trabalho consiste de uma Revisão de Literatura de artigos científicos, por meio de busca na BVS (Biblioteca Virtual em Saúde) pelos seguintes Descritores Controlados em Ciência da Saúde (DeCS): Fisioterapia; úlcera, pé diabético. Inicialmente, foram encontrados 53 artigos, sendo que 15 foram excluídos por não estarem disponibilizados com o texto na íntegra; 18 foram excluídos por serem escritos em língua estrangeira e 9 artigos foram excluídos por não serem dos últimos 5 anos. Sendo assim, foram selecionados 10 artigos para compor a presente revisão.

O DM é considerado uma doença crônica grave que ocorre quando 0 pâncreas não produz insulina suficiente ou quando o corpo não usa de forma eficaz a insulina que produz. Estudos realizados pela Organização Mundial de Saúde (OMS) estimam que o número de pessoas com diabetes na América Latina aumente para 64 milhões até $2025^{(5)}$.

Essa doença é considerada um grave problema em vários países em subdesenvolvimento, principalmente por ter um alto custo governamental para tratamento de seus portadores. Estudo 
realizado no Brasil aponta que $7,6 \%$ de indivíduos entre 30 e 69 anos são portadores de DM, sendo que $50 \%$ dessas pessoas ainda desconhecem 0 seu diagnóstico, e $25 \%$ sabem, porém não fazem nenhum tipo de tratamento(5).

Portadores de DM possuem maior risco de desenvolver úlceras do pé diabético por meio de lesões traumáticas e infecções, obedecendo a uma classificação conforme demonstrada no Quadro 1. A maior consequência negativa associada a esses problemas são as amputações $^{(6)}$.

\begin{tabular}{|c|c|}
\hline Grau da lesão & Manifestações \\
\hline Grau 0 & Pé em risco \\
\hline Grau 1 & Úlcera superficial, sem envolvimento de tecidos subjacentes. \\
\hline Grau 2 & $\begin{array}{l}\text { Úlcera profunda envolvendo músculos e ligamentos, mas } \\
\text { sem osteomielite ou abcesso. }\end{array}$ \\
\hline Grau 3 & Úlcera profunda com celulite, abcesso ou osteomielite. \\
\hline Grau 4 & Gangrena localizada (dedos, calcanhar). \\
\hline Grau 5 & Gangrena de (quase) todo o pé. \\
\hline
\end{tabular}

Fonte: Aragão (2010)(7).

Para compreender a formação das úlceras é de extrema importância saber como funciona a fisiologia do pé, que tem entre suas funções a absorção de impacto, a sustentação do corpo e proporcionar alavanca para propulsão e proteção de tecidos moles ${ }^{[8]}$ Existem fatores extrínsecos e intrínsecos relacionados a lesões do pé diabético que se associam à neuropatia periférica (NP), a doença vascular periférica (DVP) e alterações biomecânicas. Ainda se desconhece a cura para a diabetes, porém, seu controle é possível através da prevenção que pode ser realizada com atividades de autocuidados, evitando complicações, hospitalizações e mortalidade ${ }^{[5]}$.
Consultas e exames regulares se tornam uma rotina para o portador de $\mathrm{DM}^{(7)}$. A prevenção consiste em avaliação precoce, controle de fatores de risco glicêmico e cardiovascular, orientação nutricional e controle alimentar, atividades físicas e orientação do uso de sapatos e meias adequados, bem como do cuidado geral com os pés(5), incluindo a higiene, para evitar infecções fúngicas e lesões cutâneas ${ }^{(4)}$.

Atualmente, a fisioterapia não é amplamente considerada na atuação preventiva aos portadores de Diabetes Mellitus, devido às práticas fisioterapêuticas ainda centrarem no processo curativo das enfermidades ${ }^{(5)}$. Porém, a participação desse profissional 
em cuidados preventivos é de grande importância para a concretização das diretrizes de uma assistência integral à saúde, mesmo que na prática profissional em ações de promoção à saúde ainda existem muitas dúvidas sobre seu real papel $^{(5)}$.

A importância da prevenção de forma multidisciplinar ao paciente portador do DM reside da possibilidade de evitar várias complicações que podem leva-lo à incapacidade de realizar suas atividades de vida diária, sendo que problemas como pé diabético, cegueira, insuficiência renal crônica são as principais consequências negativas do DM que, além de incapacidade, levam a internações recorrentes. As deformidades osteomusculares causadas nos pés com a perda de sensibilidade podem favorecer alterações na marcha. A atuação do fisioterapeuta pode evitar esses problemas $^{(10)}$.

O paciente deve estar ciente da importância da prevenção de agravos do

\section{REFERÊNCIAS}

1. Oliveira FR. Efeito do Exercício Fìsico em Indivíduos com Diabetes: uma revisão bibliográfica. 2015; Monografia de conclusão de curso. Escola de Educação Física, Fisioterapia e Dança. Universidade Federal do Rio Grande do Sul, 48 p.

2. Lima IG de, Costa JFL, Oliveira AF, Borges JNJ, Peixoto AS, Pancieri MS, et
DM especialmente o cuidado com os pés e preocupar-se com 0 aparecimento de úlceras em extremidades inferiores, que na maioria dos casos leva a amputações ${ }^{(4)}$.

Conclui-se que o DM causa grandes impactos negativos aos seus portadores devido as suas complicações, internações de longos prazos, altos índices de incapacidade física e social relacionados a ela(5). Por isso, é fundamental a execução de ações preventivas, como educação envolvendo o indivíduo, familiares e a população como todo, bem como o diagnóstico e tratamento precoce ${ }^{(11)}$.

Os fisioterapeutas devem estar preparados para trabalhar com pacientes com DM em qualquer situação, pois a atuação da fisioterapia inicia com ações preventivas para evitar úlceras, bem como com a execução de exercícios de alongamento, fortalecimento, treino na marcha e equilíbrio, adaptações de órteses e próteses para diminuir sequelas do pé diabético, proporcionando uma melhor qualidade de vida a esses pacientes ${ }^{(12)}$.

al. Educar para prevenir: A importância da informação no cuidado do pé diabético. Rev Conex UEPG. 2017;13(1):186-95.

3. Ferreira $\mathrm{ACBH}$. Risco para desenvolver - Pé Diabético utilizando Redes Neurais Artificiais: Uma tecnologia para o Cuidado de Enfermagem. [Dissertação]. Juiz de Fora MG. Faculdade de Enfermagem. 
Universidade Federal de Juiz de Fora. 100p. 2014.

4. American Diabetes Association. Foot Complications. Living With Diabetes 2016 (cited 2018 Mar 12); Disponível em: http://www.diabetes.org/living-withdiabetes/complications/foot-complications/

5. Mendonça SS, Morais JS, Moura MCGG de. Proposta de um protocolo de avaliação fisioterapêutica para os pés de diabéticos. Fisioter em Mov 2011;24(2):285-98.

6. Andrade NHS, Dal Sasso-Mendes K, Faria HTG, Martins TA, Santos MA dos, Teixeira CR de $S$, et al. Pacientes com Diabetes Mellitus: Cuidados e Prevenção do Pé Diabético em Atenção Primária à Saúde. Rev enferm 2010;18(4):616-21.

7. Aragão ML, Fernandes VO, Quidute ARP, Sales APAM, Dantas FCM, Porto LB, et al. Perfil microbiológico e desfechos clinicos de úlceras em pés de diabéticos internados. Rev Bras Promoç Saúde. 2010;23(3):231-6 .
8. Carvalho VF, Coltro PS, Ferreira MC. Feridas em pacientes diabéticos. Rev Med (São Paulo) 2010;89 (3/4):164-9.

9. Duarte GC, Schwartz E, Santos BP dos, Lecce TM, Moura PMM. Práticas de promoção à saúde e prevenção de agravos no grupo hiperdia. Rev Espaço Ciência Saúde 2015;3:59-69.

10. Barros MFA, Mendes JC, Nascimento JA do, Carvalho AGC de. Impacto de intervenção fisioterapêutica na prevenção do pé diabético. Fisioter em Mov 2012;25(4):747-57.

11. Caiafa JS, Castro AA, Fidelis C, Santos VP, Simão E, Jr CJS. Atenção integral ao portador de Pé Diabético. J Vasc Bras 2011;10(4 sup 2):1-32.

12. Souza JM, Pedrosa RS, Oliveira FS, Oliveira ME de. Conhecimentos e atitudes dos acadêmicos concludentes de fisioterapia quanto aos cuidados preventivos no pé diabético. Rev Interdiscip 2013;6(4):124-31.

\section{Como citar (Vancouver)}

Sousa JS, Consoline NA, Anízio PD, Morsch P, Fagundes DS. A atuação da fisioterapia na prevenção de úlceras do pé diabético. Rev Cient Fac Educ e Meio Ambiente [Internet]. 2018;9(1):320-324. DOI: http://dx.doi.org/10.31072/rcf.v9i1.575 